\title{
ist \\ Una historia de la problemática ambiental y de sus efectos sobre la ecología disciplinar
}

Federico di PASQuo

\begin{abstract}
茴
RESUMEN

El objetivo principal de este artículo se encuentra orientado a elucidar, desde una perspectiva histórica, cierta influencia que la problemática ambiental tuvo sobre la ecología disciplinar. Para ello, se analizó el periodo que va de la década de 1960 hasta la actualidad. Dos resultados centrales se desprendieron del análisis propuesto y ambos se encontraron mediados por un saber ambiental que emergió junto a la problemática ambiental. El primero, constata una serie de transformaciones fenomenológicas (con el establecimiento de nuevos patrones ecológicos), epistemológicas (con la incorporación de la noción de escala y de la teoría jerárquica) y metodológicas (con la consolidación de los experimentos de mensuración) sobre la matriz de la ecología. El segundo, se vinculó con una continuidad sincrónica entre la problemática ambiental y la ecología disciplinar. Continuidad rastreada por el reconocimiento de una problemática ambiental de orden global.
\end{abstract}

Palabras-clave • Problemática ambiental. Saber ambiental. Historia de la ecología.

Ecología del paisaje. Macro-ecología.

\section{INTRODUCGIÓN}

El objetivo de este artículo se encuentra orientado a esclarecer la influencia que la problemática ambiental tuvo sobre la ecología disciplinar. Específicamente la hipótesis es que un conjunto de transformaciones dadas en el plano fenomenológico, epistemológico y metodológico de la ecología se encontraron moduladas por la aparición de la problemática ambiental. Ahora bien, por plano fenomenológico debe entenderse, una colección de regularidades en la naturaleza de interés para el ecólogo. Ala vez, el plano epistemológico implica aquí el acervo de teorías, modelos y conceptos propios de la disciplina. Y por último, el plano metodológico incluye un conjunto de operaciones de carácter experimental propios del área. Finalmente, por matriz disciplinar entiendo "la posesión común de los profesionales de una disciplina [de ciertos] elementos (...)" (Kuhn, ${ }_{1987}$ [1977], p. 321), los cuales vendrán dados por teorías, modelos y conceptos (plano epistémico), enfoques experimentales (plano metódico) y patrones, procesos y mecanismos (plano fenoménico). Para desarrollar dicha propuesta, el artículo se 
organizó en seis módulos. La próxima sección vinculada a la aparición de la problemática ambiental. La segunda sección relacionada al rol de los ecólogos frente a dicha crisis. ${ }^{1}$ La sección siguiente referida a la emergencia de cierto saber ambiental. La cuarta sección, central en el análisis propuesto, abordara las transformaciones dadas al seno de la ecología. Por último, se ofrecen algunas reflexiones históricas respecto de la relación establecida entre la ecología y la problemática ambiental.

Dicho estudio fue estructurado verticalmente, ello quiere decir que he optado por analizar ciertas transformaciones al seno de la ecología, vinculándolas con ciertos elementos extra-disciplinarios (por ejemplo, instituciones, estamentos sociales, roles y saberes, cf. Kragh, 2007 [1987], p. 113). Ello encontró su justificación, en tanto y en cuanto, dichos elementos entraron en relación con las transformaciones sugeridas en la matriz disciplinar (cf. Canguilhem, 2009 [1966], p. 22). Ese enfoque resulta alternativo a la organización horizontal de la historia, preponderante en los estudios del área (cf. Egerton, 1983, 1985; McIntosh, 1982, 1995 [1985]; Hagen, 1992; Deléage, 1993; Golley, 1993). A la vez, en algunos de esos estudios, se ha privilegiado el desarrollo interno, es decir, el desarrollo lógico de las ideas de la disciplina dejando de lado la complejidad de los acontecimientos y coyunturas en las que esas ideas encuentran un lugar (cf. Egerton, 1983; McIntosh, 1982; Real \& Brown, 1991). Por el contrario, he intentado sustituir el "tiempo lógico" de la ecología por el espesor o la densidad de un momento dado, en particular por la compleja coyuntura ofrecida por la aparición de la problemática ambiental. Asimismo, el trabajo se encuentra centrado en la historia reciente de la disciplina, desde la década de 1960 hasta la actualidad. Periodo olvidado dentro de la bibliografía canónica la cual, en general, exploró desde finales del siglo xix hasta la década de 1970 (cf. Egerton, 1983, 1985; McIntosh, 1982, 1995 [1985]; Hagen, 1992; Mitman, 1992; Deléage, 1993). Finalmente, he intentado adoptar una perspectiva discontinua de la historia, contrapuesta a la perspectiva continuista. Esta última, resultó usualmente correlativa con la idea de un sujeto fundador, donde "las revoluciones no son jamás en él otra cosa que tomas de conciencia" (Foucault, 2010 [1969], p. 24). Por el contrario, se trató aquí de la descripción de ciertas transformaciones, sin que ellas sean remitidas a una conciencia que operaría como su fundamento. En todo caso, dichas transformaciones deben aludir a un conjunto de agentes, instituciones y estamentos circunscritos en condiciones sociohistóricas determinadas.

1 Los términos "problemática ambiental” y “crisis ambiental” serán utilizados como sinónimos. A la vez, no se intentará alcanzar una definición sobre la problemática ambiental, sino mostrar a partir de que grupos sociales, estamentos e instituciones apareció cierto tema reconocido usualmente como problemática o crisis ambiental. 
UNA Historia DE LA PRoblemática AMBiental...

\section{EMERGENGIA Y DEMARGAGIÓN DE LA PROBLEMÁTICA AMBIENTAL}

\section{1 Ámbitos de emergencia}

Los ámbitos de emergencia designan "los puntos o momentos de aparición de un objeto (...) a partir de las acumulaciones sucesivas y simultáneas de prácticas, enunciados y discursos que poseen un cierto grado de coherencia" (Albano, 2004, p. 47). Como señalaré, tales enunciados y discursos vendrán dados por las manifestaciones ambientales de la década de 1960. Es a partir de ellas, donde emergió por primeras vez la problemática ambiental como objeto o tema.

En este apartado pretendo destacar únicamente a partir de que superficies primeras (grupos o sectores) se manifestó la problemática ambiental, tal que comenzó a delimitarse un dominio (o espacio de orden) para la misma. Perfilándose por primera vez, aquello de lo que se habla cuando se hace alusión a la crisis ambiental, es decir, cuando comenzó a tener la problemática ambiental "el estatuto de objeto y, por lo tanto, de hacerlo aparecer, de volverlo nominable y descriptible" (Foucault, 2010 [1969], p. 59). Son abundantes las fuentes que señalan a las primeras manifestaciones de la década de 1960 como las precursoras en anunciar una crisis ambiental. Por aquella década, una variedad de protestas representadas generalmente por jóvenes estaban "exigiendo que la naturaleza fuera tratada con respeto" (Bowler, 1998, p. 378-9). Diez años después, se manifestaba "un aumento importante en la preocupación que siente la mayoría de la gente por el medio ambiente" (Goodpaster, 2004, [1978], p. 148). Resulta interesante destacar que previamente a la aparición de las protestas ambientales, la degradación del medio fue considerada un resultado normal del progreso industrial. Dicho de otro modo, la degradación del ambiente había sido vista como una consecuencia lógica e incluso deseada del progreso humano (cf. Prado, 1996, p. 163). Fue recién a partir de aquellas primeras protestas de los sesenta, que la problemática ambiental comenzó a tener un estatuto de objeto, ${ }^{2}$ se perfiló, apareció como problema al cual había que dar respuesta. En tanto tuvo dicha visibilidad la problemática ambiental pudo ser descripta, puntualizada, explicitada por diferentes estamentos sociales e instituciones, tomando por "estamento social" a diferentes grupos sociales que comparten ciertos rasgos culturales, económicos, profesionales etc. Señalaran, en particular, en el trabajo propuesto, distintas disciplinas o áreas del conocimiento.

2 El objeto del discurso refiere al conjunto de signos que en un texto remiten a aquello de lo que se habla. Es decir, es una noción semejante a la de tema (o tópico). En este trabajo, el esfuerzo esta puesto en evidenciar la emergencia y delimitación del objeto del discurso. Es decir, bajo que condiciones apareció determinado tema (cf. Albano, 2004, p. 50). 


\subsection{Instancias DE DELIMitaGión}

A continuación, se ofrece un inventario de los distintos estamentos sociales e instituciones que designaron, nombraron e instauraron la problemática ambiental. Fue por medio de esas instancias que la crisis ambiental se delimito y aisló, adquiriendo así suficiente visibilidad (cf. Castro, 1995, p. 201). Como veremos, las instancias de delimitación ofrecieron en parte, las condiciones históricas necesarias (aunque no suficientes) para que la problemática ambiental surja como objeto de diferentes discursos, donde se reconocer conjuntos de enunciados que remiten a las mismas condiciones de aparición (cf. Castro, 2011, p. 109) que vienen dadas por las instituciones y los estamentos sociales. A la vez, dicho inventario no está dirigido a determinar la constitución interna de la problemática ambiental, es decir, a darle una definición, sino más bien, a delinear un espacio de orden, un dominio donde los discursos sobre ella emergen, se reparten, se diferencian y se depositan (Foucault, 2010 [1969], p. 63). ${ }^{3}$ Asimismo, resulta importante aclarar que las instancias de delimitación son casi simultaneas a los ámbitos de emergencia (cf. Albano, 2004, p. 48). De esa manera, luego de que la problemática ambiental adquiriera cierta visibilidad a través de los ámbitos de emergencia, se produjo su delimitación por medio de distintos estamentos sociales e instituciones.

Dado que este artículo supone la reconstrucción de cierto espacio de orden del cual la ecología fue parte, comenzaré refiriéndome a ella. En efecto, la ecología científica ha funcionado como uno de los estamentos sociales donde la crise ambiental fue delimitada, lo cual quedó reflejado

en los títulos de revistas científicas, por ejemplo, Conservation Biology, Ecology and Society, Conservation Ecology, en libros-texto como, por ejemplo, Conservation ecology, Conservation science and action, en congresos y reuniones científicas, por ejemplo, "Integración de la Ciencia de la Biodiversidad para el Bien-Estar $\mathrm{Hu}$ mano", así como en una gran cantidad de artículos, proyectos, programas de investigación, y líneas de financiación de investigaciones en ecología (Núñez et al., 2009, p. 24,2).

Otras áreas del conocimiento también han funcionado como estamentos sociales cumpliendo la misma función demarcativa, entre ellas se pueden mencionar la ética ambiental (cf. Leff, 2007), la economía ambiental o bien la economía ecológica 
(cf. Pengue, 2009), la ecología política (cf. Pengue, 2009), la educación ambiental (cf. Leff, 2007), la psicología ecológica (cf. Laplante, 2004), la antropología ecológica (véase Laplante, 2004), entre otras. Las áreas mencionadas no son los únicos ámbitos donde la problemática ambiental fue delimitada. En relación con ellas, instituciones tales como laboratorios y universidades también han demarcado la problemática ambiental. De aquí que se ha señalado un papel fundamental de las universidades y de los laboratorios en la formación del conocimiento ambiental (cf, Leff, 2007, p. 243). A su vez, en cuanto al sistema educativo en todos sus niveles, se ha indicado, por ejemplo, que resulta central en la construcción de una conciencia ambiental la cual sería clave para la aplicación de políticas ambientales exitosas (cf. Melillo, 2011, p. 8). Otras instituciones vinculadas a la problemática ambiental son los medios de comunicación, las ONGs y las empresas multinacionales. Se ha señalado, por ejemplo, que los ecologistas han utilizado los medios de comunicación "como la caja de resonancia de sus predicciones" (Prado, 1996, p. 164). O también, que muchas organizaciones no gubernamentales, dedicadas a la protección del ambiente, han adoptado modalidades muy parecidas a la de las empresas o corporaciones multinacionales denunciadas por contaminar el ambiente (cf. Latchinian, 2010, p. 56). Finalmente, se pueden sugerir dos ámbitos más, el derecho (o la justicia) y los estados. En relación con ellos, en la Declaración de Nairobi de 1982 se sugiere, por ejemplo, que "los estados deben promover el desarrollo progresivo del derecho ambiental" (Grinberg, 1999, p. 188). En este punto, se puede sintetizar lo dicho, por medio de la siguiente conclusión parcial: distintas disciplinas, universidades, laboratorios, los diferentes niveles educativos, corporaciones multinacionales, medios de comunicación, ONGs, el derecho y los estados, en tanto que instituciones o estamentos sociales, han participado, a partir de la década de 1960, en la demarcación de la problemática ambiental.

Recapitulando, la interrelación entre esas determinaciones " los ámbitos de emergencia (apartado 1.1) y las instancias de delimitación (1.2) “permitió delinear un espacio de orden, o dominio, sobre el cual pudo aparecer, ser nombrada y ser descripta la problemática ambiental. Es decir, a partir de dicho dominio ella pudo ser considerada como objeto de diferentes discursos que "determinan el haz de relaciones que el discurso debe efectuar para poder hablar de tales y cuales objetos, para poder tratarlos, nombrarlos, analizarlos, clasificarlos, explicarlos etc." (Foucault, 2010 [1969], p. 65). Es decir, los distintos estamentos e instituciones sugeridos generaron un relieve donde los discursos sobre la problemática ambiental se distribuyen y circulan. Dicho espacio determinó la dirección y el sentido de los discursos, estableció el régimen de circulación de los enunciados serios que tratan sobre la problemática ambiental, tales como reglamentos jurídicos sobre el uso de recursos naturales, informes de impacto ambiental, informes sobre manejo de fauna, informes técnicos, artículos sobre pro- 
blemas ambientales, panfletos de protesta, declaraciones ambientales, planes curriculares, documentales, libros sobre especies amenazadas, bancos de datos, entre otros. ${ }^{4}$ Paralelamente, la descripción de dicho espacio de orden esclarece porque en el periodo indagado se pudo hablar de la problemática ambiental en términos educativos, empresariales, mediáticos, políticos-económicos, legales, o bien, en términos disciplinares (sociales, humanos o naturales) (cf. Castro, 1995, p. 201).

Inventariado el dominio para la problemática ambiental, dos aspectos deben ser nuevamente destacados. El primero es que la ecología disciplinar tuvo una función demarcativa al delimitar a la problemática ambiental. El segundo es que se reconocieron otras instituciones y estamentos que también recortaron dicha problemática. A continuación, pasaré a describir los diferentes roles del ecólogo frente a esa problemática de acuerdo con el dominio sugerido.

\section{AUTORIDAD, POSIGIÓN Y GÓdIGO PERGEPTIVO DEL EGÓLOGO FRENTE A LA PROBLEMÁTICA AMBIENTAL}

\subsection{LA AUTORIDAD DEL ECÓLOGO}

En esta sección, se analiza cuál fue la autoridad del ecólogo frente a una sociedad preocupada por la crisis ambiental. Antes bien, será necesario reconocer al mismo cómo un científico perteneciente al ámbito de las ciencias denominadas "duras". Como tal, el ecólogo se ha encontrado habilitado a pronunciar todo un conjunto de enunciados vinculados al ambiente (por ejemplo, artículos, informes de impacto ambiental, informes sobre manejo de fauna etc.). Más aún, se encontró con derecho a hablar acerca de la crisis ambiental con la presunción de que lo que él dice resulta verdadero (cf. Foucault, 2010 [1969], p. 69; Pozo, 1986, p. 98; Dreyfus \& Rabinow, 1988, p. 89). El ecólogo, fue uno de los titulares que ha empleado determinado tipo de lenguaje científico tal que garantizó la veracidad de ciertos enunciados relacionados con los problemas ambientales. En ese sentido, Pengue destacó que

4. El enunciado puede ser una frase o una proposición pero siempre considerada desde el punto de vista de sus condiciones de aparición (por ejemplo, informes, panfletos, declaraciones, artículos etc.). Es decir, se entiende al enunciado como la modalidad en la que aparecen un conjunto de signos. Dicha modalidad, le permite al enunciado ser algo más que una agrupación de marcas materiales. A ello, agrega una determinación sobre los signos en el nivel de su manifestación y, de ese modo, orienta su interpretación. Por lo tanto, una misma frase manifestada bajo condiciones diferentes, puede implicar enunciados distintos (cf. Castro, 2011, p. 129). 
UNA Historia DE LA PRoblemática AMBiental...

existe la visión (...) de que (...) la palabra del experto científico es definitoria, algo aceptado generalmente en la sociedad. A pesar que en muchos casos, el científico opina, a veces, más allá de su propio campo de acción o experiencia, específicamente, en los temas ambientales. La sociedad asume que aquello que no puede ser probado científicamente, no es cierto (Pengue, 2009, p. 245).

La autoridad del científico, ese grado de aceptación o prestigio que se le concedió, vino ligada, en general, a criterios de competencia (en cuanto a los métodos y estrategias teóricas o conceptuales empleadas), a normas pedagógicas (en cuanto al tipo de formación recibida) (cf. Foucault, 2010 [1969], p. 69) y también a las condiciones institucionales en las que el ecólogo se encontró inserto (entre las que pueden destacarse laboratorios, universidades, ONGs, empresas, entre otras).

\subsection{Posiciones o ROLES DEL EGÓLOGO RESPECTO DE LA PROBlemÁticA AMBIENTAL}

Los ecólogos ocuparon distintas posiciones dentro del dominio antes descripto. Al respecto, se pueden reconocer diferentes roles o espacios de socialización " vinculados a la crisis ambiental " los cuales determinaron distintas funciones para el ecólogo. 5 Dicho de otro modo, cada una de las posiciones que éste pudo ocupar se han encontrado asociadas a un conjunto de prácticas y expectativas distintas. De tal manera, se puede sugerir el rol de investigador como una de sus funciones primeras. "La ciencia, aparte de una forma de adquirir conocimiento, es una manera de pensar y de ver la realidad (...), es una herramienta que podemos brindar como ecólogos (e investigadores en general) a la sociedad" (Gurvich et al., 2009, p. 235). A la posición de investigador ordinariamente realizada en el contexto de laboratorios y universidades se agregó el rol de técnico en tanto y en cuanto se reconoció en el ecólogo cierta capacidad de dar soluciones a problemas ambientales concretos. Por ejemplo, en relación con los problemas vinculados a las plagas de insectos, se han propuesto soluciones biológicas (alternativas al control químico) donde "están contribuyendo especialistas que representan diversos terrenos del vasto campo de la biología, a saber, entomólogos, patólogos, genetistas, fisiólogos, bioquímicos, ecólogos" (Carson, 2010 [1962], p. 292). Otras funciones que supo tener el ecólogo fue la de divulgador donde tuvo "[...] una credibilidad

5 Las comillas indican que no se considera aquí un sujeto que fuese anterior a sus roles, es decir un sujeto original o fundador: "[hay] que terminar con la ficción según la cual el sujeto, el yo sería anterior a sus roles, en tanto [que] no existe sujeto 'en estado salvaje' (...) un sujeto semejante no sería original sino vacío. No encontramos en la historia el puro sujeto de forma universal. Los sociólogos lo expresan a su manera: no existe individuo sino socializado" (Veyne, 2004, p. 65). 
alta frente a la opinión pública" (Gurvich et al., 2009, p. 235) o también la de militante ambientalista. De aquí, que muchos trabajen vinculados a diferentes aspectos de la problemática ambiental, "desarrollando una actividad creciente de divulgación, extensión, informes técnicos, trabajos en ONGs" (Gurvich et al., 2009, p. 235-6). Asimismo, se pueden destacar otros espacios de socialización que también emergieron en conjunto con la aparición de la problemática ambiental, por ejemplo, posiciones tales como la de asesor o consejero dentro de agencias gubernamentales o dentro de empresas. "Ya se dispone de gran parte del conocimiento necesario, pero no se utiliza. Formamos a ecólogos en nuestras universidades, e incluso los empleamos en nuestras agencias gubernamentales, pero rara vez aceptamos su consejo" (Carson, 2010 [1962], p. 12). Independientemente de que se acepten o no los consejos del ecólogo aquí el problema es otro, se trata simplemente de indicar esa nueva función. Ala vez, se puede sugerir un último espacio de socialización dentro del sistema educativo (cf. Leff, 2007, p. 229). En particular, en el nivel universitario, donde el ecólogo ya cumplía un rol de educador antes de la aparición de la problemática ambiental. Finalmente, cabe reconocer que las posiciones descriptas no son necesariamente excluyentes, los ecólogos "en ocasiones han desdibujado sus límites mezclando sus roles como investigador científico, como militante social y como asesor de empresas" (Latchinian, 2010, p. 88).

De cuanto he dicho, quedó establecido someramente cuales fueron los distintos roles que el ecólogo supo ocupar frente a la problemática ambiental (por ejemplo, investigador, técnico, asesor, divulgador, educador o militante-ambientalista). De tal manera, resulta interesante destacar que esa problemática en tanto que objeto del discurso se encontró, al menos en parte, "en manos" de un número de individuos que debieron ejercer determinadas funciones designadas o "reglamentadas" (cf. Dreyfus \& Rabinow, 1988, p. 87-8), y donde los ámbitos institucionales, ${ }^{6}$ tales como universidades, escuelas públicas y privadas, ONGs, empresas, medios de comunicación, laboratorios públicos y privados "brindaron el lugar" para perfilar esas diferentes posiciones o ubicaciones variables desde donde el sujeto estuvo habilitado para hablar, como muestra el diagrama 1 (cf. Deleuze, 2008 [1986], p. 36). A la vez, resulta interesante

\footnotetext{
6 Las instituciones constituyeron "un campo institucional, un conjunto de acontecimientos, de prácticas, de decisiones políticas, un encadenamiento de procesos económicos en los que figuran oscilaciones demográficas, técnicas de asistencia, necesidades de mano de obra, niveles diferentes de desempleo etc." (Foucault, 2010[1969], p. 205). Es decir, las instituciones establecieron un campo de prácticas (políticas, económicas o educativas) las cuales resultaron necesarias en el establecimiento de la autoridad y de los roles designados al ecólogo. Sin embargo, aunque se reconoce que dichas prácticas fueron necesarias, en este artículo no se analizan (cf. Habermas, 2012 [1985], p. 293; Dreyfus \& Rabinow, 1988, p. 98). Por el contrario, me focalizo en el análisis del discurso recurriendo para ello a buena parte de las herramientas del método arqueológico (cf. Gros, 2007, p. 72-3; Pardo, 1989, p. 109), el cual permitirá "componer simultaneidades o continuidades sincrónicas" (Bert, 2008 [1967], p. 37), interés central del presente artículo (cf. Bert (2008 [1967], p. 22).
} 


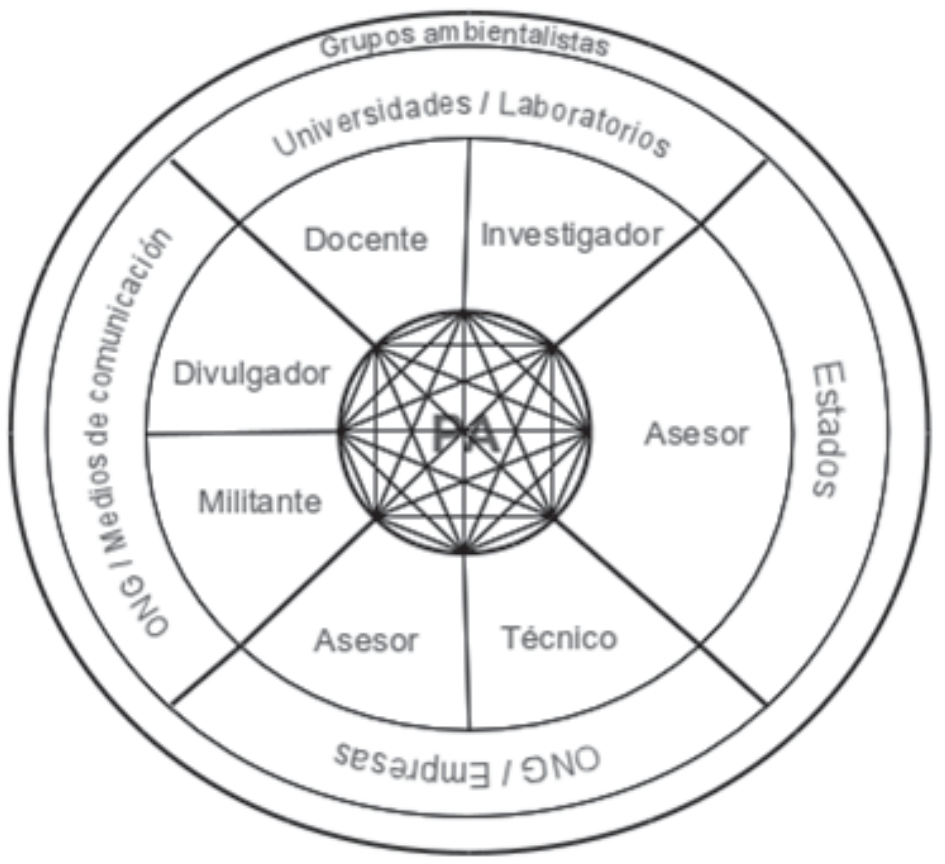

Diagrama 1. Se representa el espacio de orden sugerido. El círculo del centro corresponde al objeto del discurso, o sea, la problemática ambiental. Su entramado de líneas señala la circulación de distintos enunciados en torno al mismo. La circunferencia siguiente refiere al sujeto del discurso, el ecólogo, e indica los diferentes "roles" que emergen en dicho dominio. A la vez, la tercera circunferencia muestra las instituciones que por un lado delimitan la problemática ambiental y, por otro, hacen posibles las distintas posiciones que el ecólogo puede ocupar. Finalmente, la última circunferencia remite a los primeros sectores que dieron visibilidad a la problemática ambiental.

adelantar que antes de la crisis ambiental el rol clásico del ecólogo fue, en general, el de investigador al seno de las universidades. Sin embargo, con la imposición de un nuevo objeto del discurso y la fabricación de nuevos roles, cambiaron radicalmente las condiciones desde donde éste estuvo habilitado para hablar (cf. Gros, 2007, p. 72).

\subsection{Código Perceptivo}

En este punto, resulta elemental distinguir cierto código perceptivo, es decir, cierta codificación que constituye aquello que mira. Dicho de otro modo, este código prescribe lo que hay que ver o como debe observarse. De esa manera, se puede hablar de una mirada estructuradora hacia el objeto del discurso (cf. Albano, 2004, p. 54). La mirada estructuradora no debe ser comprendida como proveniente de un sujeto fundamental. Por el contrario, dicha mirada aparece con ciertos sujetos circunscritos a ciertas con- 
diciones sociohistóricas, tal como aquellos primeros movimientos de protesta surgidos durante la década de 1960 en determinados países industrializados. "A mediados del siglo pasado ya existían indicios de que el hombre había incidido en cambios de escala planetaria" (Latchinian, 2010, p. 25). En este sentido, se puede evidenciar cierta marca que se le ha asignado a la problemática ambiental (la cual será central a los fines de comprender una serie de transformaciones dadas al seno de la ecología disciplinar). En función de ello, destaco que, desde la década del 1960, esa mirada prescribió sobre la problemática ambiental una marca global. Dicho de otra manera, a la problemática, en tanto objeto de diferentes discursos, se le adscribió una dimensión geográfica amplia. Ahora bien, por global me refiero específicamente a una dimensión física, es decir, no debe confundirse con globalización. De manera que lo global no implica aquí contenidos económicos o ideológicos, sino más bien, se trata de poner en evidencia que simplemente se han traspasado las fronteras geopolíticas en términos espaciales, acercándose entonces a la idea de mundialización (cf. Latchinian, 2010, p. 26). En tanto que dicha marca se imprime a la problemática ambiental, se entiende, entonces, que muchos de los problemas ambientales han sido considerados sobre dimensiones geográficas amplias o si se quiere con un alcance amplio sobre la biosfera. Puede ocurrir también que una conjunción de problemas ambientales locales tengan efectos globales (cf. Latchinian, 2010, p. 26).

Existen varias listas internacionales aceptadas de problemas ambientales globales (...). Una lista bastante comprensiva podría estar integrada por (1) cambio climático y calentamiento global; (2) destrucción de la capa de ozono; (3) pérdida de biodiversidad; (4) crisis energética y crisis alimentaria; (5) contaminación de los océanos; (6) escasez y mal uso del agua; (7) degradación de suelos fértiles y desertificación; (8) destrucción de selvas y bosques tropicales; (9) lluvia ácida; (10) acumulación de desechos tóxicos (Latchinian, 2010, p. 27).

Parecería que la percepción global de la crisis ambiental se ha convertido en una formula exitosa durante el periodo de tiempo analizado (cf. Puebla, 1999, p. 26; Yustos, 2009, p. 2; Pasquo et al., 2011, p. 29). Como veremos, resulta interesante adelantar que el ecólogo y, por lo tanto, la ecología, no se encontró exento de esa mirada estructuradora.

A partir de aquí, se intentará abordar una posible relación del saber ambiental generado a partir de las determinaciones históricas consideradas, por ejemplo, ámbitos de emergencia, instancias de delimitación, autoridad, posiciones y código perceptivo, para luego evaluar el modo en que dicho saber resulto fundamental dentro de una variedad de disciplinas vinculadas a la crisis ambiental (siguiente sección) y, en particular, para la ecología disciplinar (cuarta sección). 
UNA Historia DE LA PRoblemática AMBiental...

\section{LA EMERGENGIA DEL SABER AMBIENTAL}

\section{Y SU REFORMULAGIÓN EN EL ÁMBITO DISGIPLINAR}

Habiendo podido reconocer distintas determinaciones sobre la problemática ambiental, es posible buscar ahora una caracterización de saber que las implique. En ese sentido, el saber se define aquí cómo un conjunto de elementos, o sea, objetos, nociones generales, modos de formulación, ámbitos especulativos etc., indispensables para la conformación de una ciencia, o de una disciplina, que se forman a partir de una red de relaciones y delimitaciones dadas entre:

(1) los ámbitos de emergencia, estamentos sociales e instituciones, espacio que determina el régimen de los discursos y aquellos de lo cual se puede hablar en un momento dado (cf. Foucault, 2010 [1969], p. 2378; Jarauta, 1979, p. 114; Castro, 2011, p. 363-4);

(2) la autoridad, las posiciones subjetivas y los códigos perceptivos, que determinan el espacio donde el sujeto puede ubicarse y "cómo debe mirar" (cf. Foucault, 2010 [1969], p. 237-8; Jarauta, 1979, p. 114; Castro, 2011, p. 363-4).

Según esa perspectiva, el saber queda caracterizado por la interrelación entre ámbitos de emergencia, estamentos, instituciones, autoridades, roles y códigos perceptivos. Así definido, el saber no puede agotarse en ningún estamento o institución específica ya que justamente viene (nótese, que dichos estamentos no tiene porque limitarse a disciplinas o áreas científicas) dado por las interrelaciones de un conjunto de estamentos e instituciones. Se parte, entonces, de una relación asimétrica entre el saber y una ciencia o una disciplina. Dado que el saber puede persistir con cierta independencia del desenlace de una disciplina particular, puede afirmarse que el saber no se agota en ningún ámbito disciplinar específico. Pero a la vez, ninguna disciplina emerge de novo, es decir, no se construye de cero o a partir de sus elementos más básicos. Muy por el contrario, toda ciencia o disciplina se conforma a partir de un conjunto de elementos prefabricados, propios de un saber. Por ello, puede afirmarse también que el saber es indispensable para la conformación de una ciencia dada (cf. Foucault, 2010 [1969], 238-9). Por lo demás, ese conjunto de elementos prefabricados, tal como objetos, nociones generales, modos de formulación etc. son jerárquicamente inferiores, en tanto se encuentran por debajo del nivel de cientificidad característico de una disciplina. Recuperando lo dicho, Miguel Morey mencionaba que "no todo saber da lugar a una ciencia; una ciencia determinada no reasume totalmente ni liquida el saber del que brota" (1986, p. 221). 
$\mathrm{Al}$ aplicar dicha caracterización a nuestro ámbito de estudio, se puede evidenciar la emergencia de un saber ambiental.? Éste, obliga a reconocer que el discurso de la ecología se encontró rodeado por discursos vecinos procedentes, por ejemplo, de otras áreas del conocimiento (cf. Habermas, 2012 [1985], p. 264). Lo último resulta central para este artículo. Dado que en muchos de esos discursos vecinos es posible rastrear aquella codificación, aquella marca impuesta a la problemática ambiental, pero ahora reformulada en teorías, conceptos específicos o definiciones propias de las disciplinas, y si la aparición de la problemática ambiental estuvo codificada en parte por una dimensión global, no debería llamar la atención que dicho código se encuentre reformulado en la matriz de diferentes áreas del conocimiento que delimitaron a la misma. En esa misma línea argumental, "lo que se considera 'naturaleza' en una época determinada es un producto nuestro, en el sentido de que todos los rasgos que se le adscriben han sido primero inventados por nosotros y usados después para otorgar orden a lo que nos rodea" (Feyerabend, 1982 [1962], p. 4.0). Ello implica, entonces, que dicha marca no sólo actuó como un código perceptivo (apartado 2.3), sino que también se desempeño como un elemento del saber ambiental, relevante en la conformación de distintas disciplinas. Dicho de otra manera, la dimensión geográfica global tuvo una doble función: como código perceptivo en la construcción del objeto del discurso y cómo elemento del saber ambiental, en tanto fue una noción reactualizada por diferentes áreas del conocimiento. Para evidenciar esa segunda función se pueden mencionar, a modo de ejemplos, (1) el tema de la "difusibilidad" dentro del derecho ambiental internacional, (2) el concepto de "calidad de vida" dentro de la ética ambiental y (3) una definición dada de la ecología política.

$\mathrm{El}$ asunto de la difusibilidad parte del reconocimiento de que muchos recursos naturales comprenden grandes regiones geográficas y atraviesan más de una nación. Así, al intentar reglamentar jurídicamente la titularidad sobre ese recurso natural transnacional se presenta el problema de supervisar su utilización: "la inteligibilidad y codificación de los nuevos derechos se enfrenta al problema de su difusibilidad, es decir, a la dificultad de definir la titularidad de los bienes comunes y crear una ingeniería jurídica para normar y supervisar sus usos" (Leff, 2007 , p. 127-8).

La reflexión en torno al concepto de "calidad de vida" vino a reemplazar y a problematizar el concepto de "mejor nivel de vida". En relación a ello, ciertas posturas sostienen que el primer concepto puede utilizarse como una guía para reflexionar so-

${ }_{7}$ Enrique Leff menciona al respecto: "las perspectivas foucaultianas sobre el saber (...) nos permiten ver la irrupción del saber ambiental [constituido entre otras cosas por] el discurso y las prácticas del movimiento ambientalista, por el discurso oficial del estado y por el ordenamiento jurídico de la legislación ambiental. Desde allí es posible aprehender al saber ambiental desde (...) su inserción en diferentes dominios institucionales y campos de aplicación” (Leff, 1994, p. 49-50; cf. Leff, 2000 [1986], p. 27-87; 2009, p. 232-3). 
bre una ética planetaria "pues por primera vez el hombre ha accedido a una toma de conciencia global de la especie humana (...). Del hecho de esa toma de conciencia global nace la necesidad de elaborar una tabla de valores, una ética, de dimensiones planetarias" (Blanch apud Leff, 2007, p. 114). Dejando a un lado los contenidos valorativos resulta interesante destacar que la idea de una nueva conciencia presupone una dimensión geográfica de carácter global.

Según Héctor Alimonda, la ecología política puede definirse como el estudio de las articulaciones entre prácticas y representaciones "a través de los cuales diferentes actores políticos, actuantes en diferentes escalas (local, regional, nacional, internacional) se hacen presentes (...) en la constitución de territorios y en la gestión de su dotación de recursos naturales" (Alimonda 2011, p. 44). Nuevamente se hace patente aquella dimensión global puramente espacial en la definición sugerida.

En cada una de esas áreas se puede reconocer, una reactualización o reformulación de la dimensión global de la problemática ambiental (ver diagrama 2). Poniéndose en evidencia que dicha noción resultó ser, al menos en parte, un elemento del saber ambiental relevante en la conformación de dichas áreas.

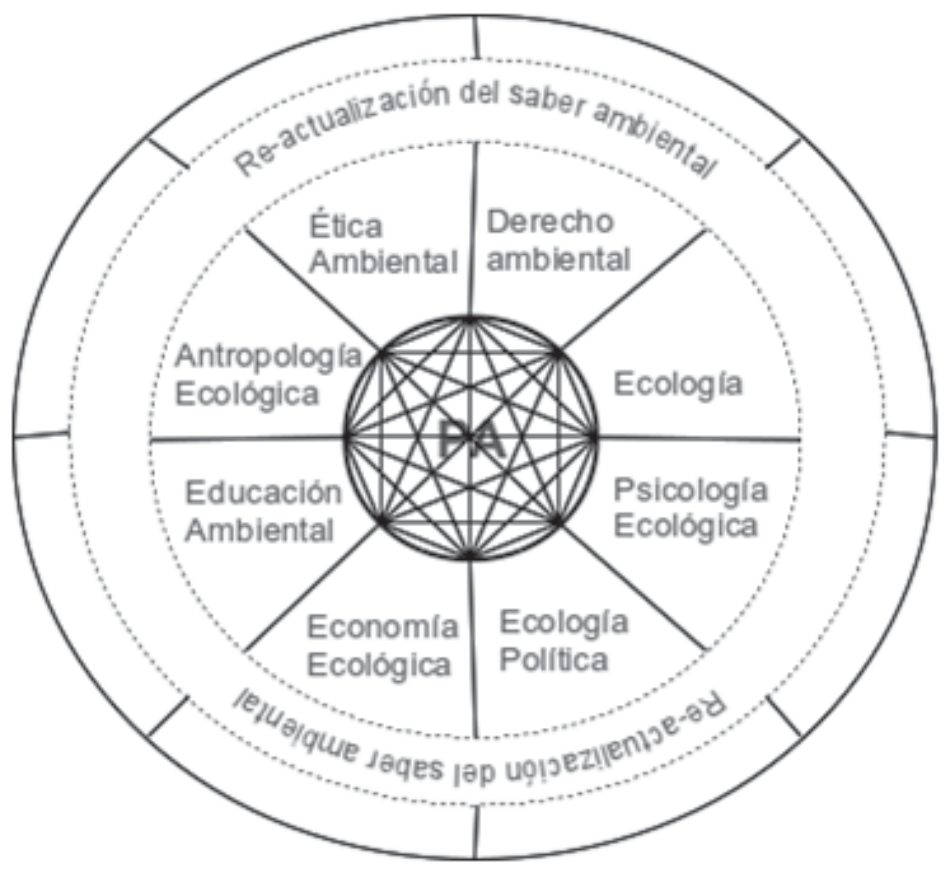

DiAgRAMA 2. Nuevamente el círculo del centro corresponde al objeto del discurso, o sea, la problemática ambiental y su entramado de líneas señala la circulación de distintos enunciados en torno al mismo. La circunferencia siguiente indica ahora a distintas disciplinas "vecinas", las cuales, por un lado, han delimitado a la problemática ambiental y, por otro, han reactualizado ciertos elementos del saber ambiental (por ejemplo, la dimensión geográfica global). 
Esa misma reformulación del saber ambiental mediada por la dimensión global también tuvo lugar en la ecología disciplinar. Es decir, dicho elemento resultó indispensable en la conformación de dos subáreas de la ecología (la ecología del paisaje y la macroecología). Para alcanzar la forma global dentro de las investigaciones ecológicas fueron necesarias una serie de transformaciones fenomenológicas, epistemológicas y metodológicas, afectándose así la matriz disciplinar de dicha área del conocimiento.

\section{TRANSFORMAGIONES FENOMENOLÓGICAS, EPISTEMOLÓGIGAS Y METODOLÓGIGAS EN LA EGOLOGÍA DISGIPLINAR: HAGIA LA FORMA GLOBAL}

\subsection{SingularizaGión DEL Discurso DE LA EGOLOGÍA}

Llegados a este punto es importante precisar ciertos filtros conceptuales por medio de los cuales, la ecología se aproximó a un problema ambiental dado y, en general, al estudio del ambiente. Hasta el momento, me he referido a la emergencia y a la delimitación de la problemática ambiental, donde señalé que la ecología funcionó como uno de los estamentos sociales que demarcó a la misma (primera sección). Aquí, resulta interesante agregar a tal demarcación, las herramientas conceptuales que la ecología tuvo para realizar sus investigaciones (cf. Albano, 2004, p. 48). Alos fines del artículo interesa destacar una primera distinción reconocida dentro de la ecología (y fuera de la misma): los fenómenos ecológicos no son los fenómenos ambientales. Esa división resulta central para diferenciar la ecología como área de la biología de otras disciplinas también dedicadas a lo ambiental como, por ejemplo, la ética ambiental, la educación ambiental, la ecología política, la antropología ecológica etc. Así el fenómeno ambiental es aquel que perturba el desarrollo normal de una sociedad y, a la vez, se encontró asociado en general a las actividades del hombre que degradan el ambiente. Mientras que el fenómeno ecológico representó un obstáculo para el conocimiento científico ecológico y se encontró vinculado más bien a regularidades en la naturaleza de interés para el ecólogo. De ese modo, mientras la ecología focalizó sobre patrones, procesos y mecanismos ecológicos a partir de fenómenos ecológicos, ${ }^{\mathbf{8}}$ muchas otras disciplinas se focalizaron sobre fenómenos que estorban el desarrollo normal de una sociedad

8 El patrón fue entendido como eventos repetidos, entidades recurrentes, relaciones replicadas o trayectorias regulares e irregulares registradas en el espacio y tiempo. En cuanto al proceso ecológico, se lo definió como un conjunto de fenómenos en donde los acontecimientos se suceden en el espacio y en el tiempo (dichos fenómenos pueden estar o no relacionados causalmente). Finalmente, el mecanismo fue concebido como un tipo especial de proceso en donde un conjunto de causas que refieren a una interacción directa se traduce en un fenómeno (cf. Pickett et al., 2007, p. 69). 
(cf. Pasquo et al., 2011, p. 23; Passmore, 1978, p. 61-2). Se puede agregar que muchos problemas ambientales resultan ser, en parte, problemas ecológicos, por ejemplo, la fragmentación de hábitats por actividad humana puede resultar en una consecuente disminución de la diversidad de especies. De cuanto he mencionado se puede sugerir que si bien los problemas de la ecología y los problemas ambientales se pueden encontrar relacionados, ellos son de distinto tipo. De aquí que se reconozca que los problemas ambientales no son necesariamente problemas científicos ecológicos (cf. Prado, 1996, p. 164).

Habiendo reconocido algunas de las principales herramientas (tales como las nociones de fenómeno, patrón, proceso y mecanismo) por medio de las cuales la ecología disciplinar se aproximó al estudio del ambiente, se puede evaluar cómo la noción antes considerada - "la dimensión geográfica global" - resultó un elemento central en la conformación de dos sub-áreas de la disciplina, a saber, la ecología del paisaje y la macroecología. De ese modo, la emergencia de estas subáreas (la primera durante la década de 1960 y la segunda a fines de la década de 1980) vinieron acompañadas por una serie de transformaciones (en el plano fenoménico, epistémico y metódico) que permitieron incorporar dicho elemento del saber ambiental (la dimensión global) al costo de tener que modificar la matriz disciplinar.

\subsection{TransformaGiones FENOMÉNICAS, EPISTÉMICAS \\ Y METÓDICAS DE LA MATRIZ DISGIPLINAR}

\subsubsection{Establecimiento DE NUEVos PATRONES EGOlóGicos A ESCALAS AMPLIAS}

Uno de los ejes centrales de la ecología del paisaje, estuvo orientado a determinar de qué manera el patrón de paisaje tiene efecto sobre los procesos y los mecanismos ecológicos y cómo éstos, a su vez, modifican dicho patrón. Este último fue usualmente representado por medio de modelos que buscan caracterizar la heterogeneidad sobre el terreno. Por otro lado, la macroecología se encontró dirigida al análisis de patrones de distribución estadística, donde se comparan especies similares en su taxonomía o en su ecología (utilizando variables diferentes). Se supone que las formas y los límites de las distribuciones estadísticas analizadas reflejan la acción de procesos y restricciones evolutivas o ambientales. Por lo tanto, la emergencia de esas dos subáreas estuvieron asociadas al reconocimiento de nuevos patrones ecológicos. Resulta interesante destacar que, a pesar de las diferencias entre los patrones analizados por cada subdisciplina - los primeros referidos a patrones espaciales sobre el terreno y los segundos a patrones de distribución estadística - ellos tuvieron en común que se encontraron generalmente ligados a grandes dimensiones físicas, es decir, son patrones 
ecológicos que descansan sobre dimensiones geográficas amplias. Así, en el ámbito de la ecología del paisaje, Mónica Turner y colaboradores destacan que "nuevas técnicas de investigación son requeridas en la ecología del paisaje debido al foco sobre el patrón espacial y la dinámica sobre grandes áreas" (Turner et al., 2001, p. 9), que también "la ciencia de la ecología del paisaje es especialmente dependiente de datos de alta calidad, ya que a menudo se enfoca en patrones y procesos a gran escala y referidos a largo plazo" (Iverson, 2007, p. 11). De ese modo la ecología del paisaje, al focalizarse en el estudio de áreas geográficas amplias, indagó patrones sobre dimensiones físicas grandes (cf. Turner et al., 2001, p. 2-3; Bogaert \& Hong, 2004, p. 109). En cuanto al ámbito de la macroecología se destacó que "un enfoque macroscópico (...) puede identificar patrones de abundancia, distribución y diversidad en escalas temporales y espaciales mucho más amplias que los de estudios ecológicos tradicionales" (Brown, 2003, p. 47), o bien que "usamos el prefijo 'macro' (...) con objeto de caracterizar patrones en las distribuciones estadísticas [que] con frecuencia es necesario trabajar a escalas espaciales y/o temporales relativamente grandes para obtener muestras suficientemente grandes" (Brown, 2003, p. 25), de modo tal que la macroecología se caracterizó por analizar patrones estadísticos entre diferentes variables que al igual que los patrones de paisaje estuvieron, en general, asociados a dimensiones geográficas amplias. De aquí que se evidencie en ambos casos una reactualización del saber ambiental mediado por la dimensión global.

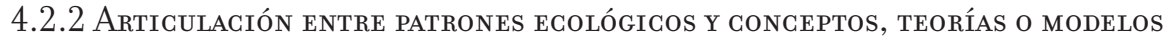

La incorporación de nuevos patrones a la ecología (provenientes, por ejemplo, de la ecología del paisaje o de la macroecología) estuvo acompañada de nuevas estrategias teóricas, modelos y conceptos claves que posibilitaron diferentes explicaciones. Se destacaron especialmente la noción de escala espacio-temporal y la teoría jerárquica, incorporadas a la ecología a finales de la década del 1970 y comienzos de 1980 (cf. Schneider, 2001, p. 552). La repercusión de la noción de escala fue tan importante que la misma fue incorporada a todas las subáreas de la ecología (cf. Wu, 2007, p. 115). En cuanto a la teoría jerárquica íntimamente relacionada a la noción de escala (cf. Turner et al., 2001, p. 34), se encontró, en general, más vinculada al ámbito de la ecología del paisaje.

La escala fue definida como "la dimensión espacial o temporal de un objeto o de un proceso" (Turner et al., 2001, p. 3). A partir de ese concepto se destacó que los patrones en ecología y dicha noción están fuertemente articulados. "La descripción del patrón es la descripción de una variación, y la cuantificación de la variación requiere la determinación de una escala" (Levin, 1992, p. 1947). A la vez, se reconoció que los 
procesos y los mecanismos suelen ser preponderantes a una escala (o en un conjunto de escalas) mientras que los patrones ecológicos pueden ser dependientes de la misma (cf. Jaksic \& Marone, 2007, p. 202). Además, se reconocen también patrones independientes de la escala, por ejemplo, los patrones fractales (cf. Brown, 2003, p. 125). Por ello, el "supuesto de fondo (...) es que muchos, si no la mayoría, de los patrones y procesos tienen lugar sobre un intervalo finito de escalas (o dominios de escala) y, por lo tanto, fenómenos diferentes pueden ser caracterizados por un dominio de escala distintivo" (Wu, 2007, p. 119). Así, mediante la incorporación del concepto de escala, ciertas posiciones dominantes dentro de la ecología, sostienen que las investigaciones deben focalizarse sobre la escala a la cual actúa el fenómeno de interés (y los procesos o mecanismos que de él pueden derivarse) (cf. Wu, 2007, p. 119).

Respecto de la teoría jerárquica, se ha señalado como una de sus principales predicciones cierta correlación entre las escalas espaciales y temporales. "Los fenómenos que ocurren sobre un área grande son más lentos que los que ocurren sobre un área pequeña” (Burel \& Baudry, 2004, p. 31). En dicha propuesta, también se destacó que, para estudiar los procesos que tienen lugar en un dominio de escalas distintivo, es necesario considerar los patrones de un dominio de escalas inmediatamente superior y los procesos de un dominio de escalas inmediatamente inferior, ya que los primeros pueden funcionar limitando el rango de las velocidades de los procesos estudiados y los segundos pueden actuar como los mecanismos que explican la dinámica de esos procesos (cf. Turner et al., 2001, p. 9; Wiens et al. 2004, p. 29). Otras propuestas teóricas también han aparecido con el fin de articularse con aquellos patrones que se vinculan a grandes regiones geográficas. En la ecología del paisaje, se puede destacar el modelo de parche-corredor-matriz el cual fue ampliamente reconocido por su simplicidad para detectar patrones de paisaje (cf. Forman, 1995, p. 135). En cuanto a la articulación entre patrones macroecológicos y estrategias teóricas, se puede mencionar, por ejemplo, los modelos de restricciones energéticas (Brown, 1999, p. 8; 2003, p. 151-2).

Las estrategias teóricas incorporadas no solo debieron articularse con los patrones ecológicos a los que refieren, sino también debieron articularse con otras propuestas teóricas dentro de la ecología y de la biología en general. En ese sentido, por ejemplo, se mencionó que el modelo de paisaje parche-corredor-matriz viene a ser una extensión del modelo de islas (cf. Lindenmayer, 2009, p. 20), o también que la teoría metapoblacional viene a indagar la unidad demográfica del paisaje (cf. Opdam, 1991, p. 94-5). A su vez, en relación a la macroecología, se ha destacado cierta relación con la teoría evolutiva (cf. Sterelny, 2005, p. 319)

En este punto, es necesario detenerse y destacar que en el concepto de escala, es decir, en la dimensión física de un objeto o de un proceso ecológico, se reformula, al menos en parte, aquella dimensión global del saber ambiental. Así, es a partir de dicho 
concepto y de la teoría jerárquica asociada que los procesos, los patrones y los objetos abordados por la ecología descansaron sobre una variedad de dimensiones físicas que van desde las más pequeñas (escalas locales) hasta las más grandes (escalas regionales, continentales o globales).

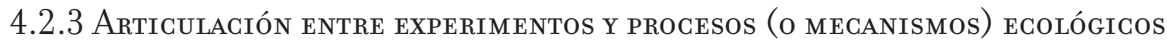

El reconocimiento de patrones y procesos ecológicos vinculados a grandes dimensiones físicas, también estuvo acompañado por una reestructuración del plano metodológico. Pues se cuestionó como manipular experimentalmente procesos o mecanismos que actúan sobre escalas espaciales amplias. Ellos, al estar asociados a un conjunto específico de escalas (apartado 4.2.2), determinan la o las escalas donde debe conducirse el diseño experimental:

el conjunto de escalas sobre las cuales un mecanismo (o conjunto de mecanismos) es válido determina directamente la escala adecuada, espacial [y] temporal (...) para las pruebas experimentales críticas de los mecanismos en cuestión (Dunham \& Beaupre, 1998, p. 36).

Por lo tanto, si se quiere manipular experimentalmente un proceso (o un mecanismo) que actúa en dimensiones físicas amplias, se debe elaborar un diseño experimental que respete ese conjunto de escalas distintivo. Dicho asunto puso en evidencia la imposibilidad de realizar tales manipulaciones, es decir, se reconoció que resulta impracticable manipular la naturaleza sobre dimensiones físicas grandes (cf. Odum \& Barrett, 2008 [1953], p. 488). Dicho de otro modo, el clásico experimento controlado es inviable sobre escalas espaciales amplias. Ese obstáculo se destacó sobre todo en aquellas subdisciplinas de la ecología que indagaron patrones (y procesos o mecanismos asociados) que descansan sobre grandes regiones geográficas, como ocurre en la ecología del paisaje y la macroecología (cf. Pasquo, 2012; Pasquo \& Folguera, 2012).

La inviabilidad de la experimentación controlada propició la consolidación de otra clase de metodología, susceptible de ser aplicada sobre grandes dimensiones físicas, o sea, los experimentos de mensuración o observacionales. Adiferencia del clásico experimento, caracterizado por el control, los tratamientos, las replicas etc., en éstos se buscó, en general, comparar dos sitios distintos - uno de los cuales funcionó como el control - antes y después de una perturbación determinada (cf. Miao et al., 2009, p. 6).9

9 En este artículo alcanza con mencionar esa primera gran división de manipulación-mensuración. Para profundizar el tema, cf. Eberhardt \& Thomas, 1991. 
Dicha perturbación puede ser controlada (donde el ecólogo modifica el sistema de estudio) o bien, no controlada, por ejemplo, cuando ocurre un accidente natural o antrópico. En otras palabras, los ecólogos no intervienen manipulando el sistema de interés en todo caso, solo buscan controlar los procesos de observación (cf. Eberhardt \& Thomas, 1991, p. 54). Resalto, entretanto, que dicha aproximación puede aplicarse a grandes regiones geográficas. Resulta interesante destacar que los experimentos de mensuración se han consolidado simultáneamente a la emergencia de la ecología del paisaje en la década de 1970 y a la macroecología en la década de 1990. En el contexto de la primera, se destacó que

los experimentos de mensuración ofrecen un medio de superar algunas de las limitaciones importantes que nosotros hemos discutido para los experimentos manipulativos. Lo más importante, las dificultades prácticas y logísticas de implementar tratamientos a grandes escalas son evitados totalmente (...). Para muchas preguntas sobre fragmentación, debido a problemas de escala y alcance, los experimentos de mensuración son los únicos enfoques viables (Mcgarigal \& Cushman, 2002, p. 338-9).

En el ámbito de la macroecología se reconoce que los experimentos de mensuración "son probablemente las únicas formas en los cuales los experimentos pueden ser realizados en escalas espaciales y temporales de interés para los macroecólogos" (Blackburn, 2004, p. 404).

\section{ConsideraGiones FINALES}

De cuanto he dicho, se puede reconocer una reestructuración de la matriz disciplinar de la ecología mediada por aquel elemento del saber sugerido, la dimensión geográfica global. Las transformaciones mencionadas han reactualizado dicho elemento, que es esencial en la conformación tanto de la ecología del paisaje como de la macroecología. Así, ese elemento se encontró en el centro de las transformaciones tanto del plano fenomenológico, con la incorporación de nuevos patrones a gran escala, como del plano epistemológico, con la incorporación y articulación de estrategias teóricas que dieran cuenta de aquellos patrones a gran escala, y también del plano metodológico, con la consolidación de los experimentos de mensuración en las investigaciones dirigidas a testear procesos sobre grandes dimensiones físicas. Las transformaciones de la matriz disciplinar sugeridas permiten señalar una ruptura en el desarrollo del área. Antes de que la problemática ambiental emergiera en la década de 1960, la ecología dirigía 
sus investigaciones sobre dimensiones físicas familiares al ecólogo (cf. Wiens, 1989, p. 385). Dicha tendencia se mantuvo incluso hasta la década de 1980, momento en que se consolidaba la ecología del paisaje y emergía la macroecología (cf. Pasquo et al., 2011, p. 32). Por aquella época, se privilegiaron investigaciones conducidas sobre dimensiones físicas pequeñas, caracterizadas por: (1) patrones vinculados a escalas finas; (2) teorías, conceptos y modelos dirigidos a explicar esos patrones, tales como, competencia, predación, polinización, parasitismo, relaciones tróficas etc. (cf. Jaksic \& Marone, 2007, p. 205); y (3) experimentos manipulativos, en condiciones de laboratorio o de campo (cf. Pasquo, 2012, p. 28-9).

A partir de la problemática ambiental y con la mediación de cierto saber ambiental, la ecología modificó aquella tendencia dirigiendo investigaciones sobre una variedad de dimensiones físicas. Dichas investigaciones estuvieron caracterizadas por: (1) patrones vinculados tanto a escalas pequeñas como grandes; (2) teorías, conceptos y modelos dirigidos a explicar esos patrones a múltiples escalas, tales como, la teoría jerárquica, el concepto de escala, el modelo de parche-corredor-matriz o los modelos de restricciones energéticas; y (3) dos clases de experimentos, los manipulativos circunscritos a escalas pequeñas y los de mensuración conducidos a escalas amplias (Pasquo \& Folguera, 2012, p. 212).

En relación a lo antes dicho, se destaca que la ruptura señalada no se vinculó con una renovación total de la matriz disciplinar, donde diferentes elementos epistemológicos, metodológicos y fenomenológicos fueron abandonados y reemplazados por otros. Por el contrario, dicha ruptura se relacionó más con la incorporación de nuevos fenómenos y estrategias teóricas y a la consolidación de ciertas aproximaciones experimentales que obligaron a cierta reestructuración, generando ese antes y después de la problemática ambiental.

¿Cómo debe comprenderse la influencia dada por la coyuntura de la problemática ambiental sobre la ecología disciplinar? Para evidenciar dicha influencia antes, fue necesario reconocer una serie de "puntos de contacto" entre la problemática ambiental y la ecología. Puntos que emergieron y se volvieron inteligibles en tanto que apareció la problemática ambiental con su dominio asociado. Acorde con esto, se destacó a la ecología como estamento social que delimito esa problemática, a la autoridad del ecólogo frente a la mirada del público no especializado, a las posiciones del ecólogo frente a los problemas ambientales, tales como, investigador, técnico, asesor, divulgador, educador o militante-ambientalista, al código perceptivo inscripto sobre la problemática ambiental, ampliamente compartido por diferentes disciplinas en tanto que reactualizan un saber ambiental. Todos eses puntos de contacto funcionaron como "poros" por medio de los cuales la coyuntura dada por la problemática ambiental y la ma- 
triz de la ecología se acercaron recíprocamente. Así, fue posible reconocer una serie de enunciados - en sus diferentes modalidades, por ejemplo, artículos académicos, declaraciones, informes de impacto ambiental, panfletos de protesta etc. - , donde la ecología y la problemática ambiental se fusionaron. Por ello es que las transformaciones sugeridas sobre la matriz disciplinar, tanto fenomenológicas, epistemológicas y metodológicas, como la emergencia de las dos subáreas consideradas, la ecología del paisaje y la macroecología, pueden ser ensambladas, al menos en parte, como los efectos de una coyuntura dada por la aparición de la problemática ambiental.

Finalmente, cabe mencionar que la organización vertical del artículo habilitó simultáneamente un doble análisis, con dos resultados importantes. Por un lado, el periodo de tiempo elegido, de la década de 1960 hasta la actualidad, fue clave para poder evidenciar las transformaciones descriptas en la matriz de la ecología. Resulta que se puede, reconocer en términos históricos, un primer resultado, una discontinuidad diacrónica al seno del área, un antes y un después de la problemática ambiental reflejado en la matriz de la ecología. A su vez, fue posible establecer un segundo resultado, un encadenamiento, una continuidad sincrónica, un hilo conductor que nació con cierto código sobre la problemática ambiental (la dimensión global) y que se hizo patente en diferentes listas de problemas ambientales. Código que se recuperó como un elemento del saber ambiental, reformulado por diferentes áreas del conocimiento tales como el derecho ambiental, la ecología política, la ética ambiental y, enfáticamente, en la ecología disciplinar. Reactualización de un saber ambiental que se hizo visible en cuanto se siguió el rastro de lo global.

Federico di PAsquo Consejo Nacional de Investigaciones Científicas y Técnicas, Universidad de Buenos Aires, Argentina. dipasquof@yahoo.com.ar

\section{A history of the environmental problematic and its effects on the discipline of ecology}




\begin{abstract}
The main objective of this article is to elucidate, from a historical perspective, the influence that environmental issues have had on the discipline of ecology. To this end, the period from the 1960 s to the present was examined. The proposed analysis revealed two main results; both were found to be mediated by environmental knowledge that emerged along with environmental issues. The first refers to a series of phenomenological (establishing new ecological patterns), epistemological (incorporating the notion of scale and hierarchical theory) and methodological (consolidating of experiments involving measurements) transformations over the ecology matrix. The second was linked with a synchronic continuity between environmental issues and the discipline of ecology. This continuity was traced by the recognition that environmental issues have global impact.
\end{abstract}

KEYwords $\bullet$ Environmental problematic. Environmental knowledge. History of ecology. Landscape ecology. Macro-ecology.

\title{
REFERENGIAS BIBLIOGRÁFICAS
}

Albano, S. Michel Foucault. Glosario epistemológico. Buenos Aires: Cuadrata, 2004.

Alimonda, H. (Ed.). La colonización de la naturaleza. Buenos Aires: Clacso, 2011.

. La colonialidad de la naturaleza. Una aproximación a la ecología política latinoamericana. In:

Alimonda, H. (Ed.). La colonización de la naturaleza. Buenos Aires: Clacso, 2011. p. 19-59.

Bert, J. F. Diálogo Raymond Aron-Michel Foucault. Buenos Aires: Nueva Visión, 2008 [1967].

Blackburn, T. M. Method in macroecology. Basic and Applied Ecology, 5, 5, p. 4.01-12, 2004.

Bogaert, J. \& Hong, S. Landscape ecology: monitoring landscape dynamics using spatial pattern metrics. In: Hong, S. et al. (Ed.). Ecological issues in a changing world. Status, response and strategy. The Netherlands: Kluwer Academic, 2004. p. 109-32.

Bowler, P. J. Historia fontana de las ciencias ambientales. México: Fondo de Cultura Económica, 1998.

Brown, J. H. Macroecology: progress and prospect. Oikos, 87, p. 3-14, 1999. Macroecología. México: Fondo de Cultura Económica, 2003.

Bured, F. \& BAudry, J. Landscape ecology concepts, methods and applications. New York: Science Publishers, 2004 .

Canguilhem, G. Estudios de historia y de filosofía de las ciencias. Buenos Aires: Amorrortu, 2009 [1966].

CARson, R. Primavera silenciosa. Barcelona: Crítica, 2010 [1962].

CAstro, E. Pensar a Foucault: interrogantes filosóficos de La arqueología del saber. Buenos Aires: Biblos, 1995. . Diccionario de Foucault. Temas, conceptos y autores. Buenos Aires: Siglo Veintiuno, 2011.

Cuddington, K. \& Beisner, B. E. (Ed.). Ecological paradigms lost. Routes of theory change. New York: Elsevier Academic Press, 2005.

DelÉAGe, J. P. Historia de la ecología. España: Icaria, 1993.

Deleuze, G. Foucault. Buenos Aires: Paidós, 2008 [1986].

Dreyfus, H. L. \& Rabinow, P. Michel Foucault. Más allá del estructuralismo y la hermenéutica. México: Universidad Nacional Autónoma de México, 1988.

Dunham, A. E. \& BeAupre, S. J. Ecological experiments: scale, phenomenology, mechanism and the illusion of generality. In: Resetarits, W. J. Jr. \& Bernardo, J. (Ed.). Experimental ecology. Issues and perspectives. Oxford: Oxford University Press, 1998. p. 27-49.

Eberhardt, L. L. \& Thomas, J. M. Designing environmental field studies. Ecological Monographs, 61, 1, p. $5^{3-} 7^{3,} 1991$. 
UNA Historia DE LA PRoBlemática AMBiental...

Egerton, F. N. The history of ecology: achievements and opportunities, part one. Journal of the History of Biology, 16, 2, p. 259-310, 1983.

The history of ecology: achievements and opportunities, part two. Journal of the History of Biology, 18,1 , p. $103-4,3,1985$.

Eribon, D. (Ed.). El infrecuentable Michel Foucault. Buenos Aires: Letra Viva, 2004.

Feyerabend, P. K. Límites de la ciencia. Explicación, reducción y empirismo. Barcelona: Paidós, 1982 [1962].

Foucault, M. La arqueología del saber. Buenos Aires: Siglo Veintiuno, 2010 [1969].

Forman, R. T. T. Some general principles of landscape and regional ecology. Landscape Ecology, 10, 3, p. $133-42,1995$.

Golley, F. B. A history of the ecosystem concept in ecology: more than the sum of the parts. New Haven/London: Yale University Press, 1993.

GoodPAster, K. E. Sobre lo que merece consideración moral. In: VALDÉs, M. M. (Ed.). Naturaleza y valor. Una aproximación a la ética ambiental. México: Fondo de Cultura Económica, 2004, [1978]. p. 147-68.

Grinberg, M. Ecofalacias. Buenos Aires: Galerna, 1999.

Gros, F. Michel Foucault. Buenos Aires: Amorrortu, 2007.

Gurvich, D. E. et al. El rol del ecólogo ante la crisis ambiental actual. Ecología Austral, 19, p. 233-8, 2009.

Habermas, J. El discurso filosófico de la modernidad. Madrid: Katz, 2012 [1985].

HaGen, J. B. An entangled bank: the origins of ecosystem ecology. New Brunswick: Rutgers University Press, 1992.

Hong, S. et al. (Ed.). Ecological issues in a changing world. Status, response and strategy. The Netherlands: Kluwer Academic Publishers, 2004.

IvERSON, L. R. Adequate data of known accuracy are critical to advancing the field of landscape ecology. In: Wu, J. \& Новвs, R. J. (Ed.). Key topics in landscape ecology. Cambridge: Cambridge University Press, 2007. p. 11-38.

Jaksic, F. \& Marone, L. Ecología de comunidades. Santiago: Universidad Católica de Chile, 2007.

Jarauta, F. La filosofía y su otro. Valencia: Pre-Textos, 1979.

KraGh, H. Introducción a la historiografía de la ciencia. Barcelona: Crítica, 2007 [1987].

Kunn, T. S. La tensión esencial. México: Fondo de Cultura Económica, 1987 [1977].

Latchinian, A. Globotomía. Garacas: Puntocero, 2010.

LAPLANTE, K. de. Toward a more expansive conception of ecological science. Biology and Philosophy, 19, 2, p. 263-281, 2004.

LefF, E. (Ed.). Ciencias sociales y formación ambiental. Madrid: Gedisa, 1994. Sociología y ambiente: formación socioeconómica, racionalidad ambiental y transformaciones del conocimiento. In: Leff, E. (Ed.). Ciencias sociales y formación ambiental. Madrid: Gedisa, 1994 . p. $17^{-84}$.

. Los problemas del conocimiento y la perspectiva ambiental del desarrollo. México: Siglo Veintiuno, 2000 [1986].

Ambiente y articulación de ciencias. In: LeFF, E. (Ed.). Los problemas del conocimiento y la perspectiva ambiental del desarrollo. México: Siglo Veintiuno, 2000 [1986]. p. 27-87.

Saber ambiental. México: Siglo Veintiuno, 2007.

Racionalidad ambiental. México: Siglo Veintiuno, 2009.

Levin, S. A. The problem of pattern and scale in ecology. Ecology, 73, 6, p. 1943-67, 1992.

Lindenmayer, D. B. Large-scale landscape experiments. Cambridge: Cambridge University Press, 2009.

Liu, J. \& TAYLOR, W. W. (Ed.). Integrating landscape ecology into natural resource management. Cambridge:

Cambridge University Press, 2004.

Mggarigal, K. \& Cushman, S. A. Comparative evaluation of experimental approaches to the study of habitat fragmentation effects. Ecological Applications, 12, 2, p. 335-45, 2002. 
MaIntosh, R. P. The background and some current problems of theoretical ecology. In: SaARinen, E. (Ed.). Conceptual issues in ecology. Boston: Dordrecht Reidel, 1982. p. 1-61.

. The background of ecology. Concept and theory. Cambridge: Cambridge University Press, 1995 [1985].

Melillo, F. Educación ambiental. Ideas y propuestas para docentes. Buenos Aires: Ministerio de Educación de la Nación y Fundación Educambiente, 2011.

MiaO, S. et al. Introduction. Unprecedented challenges in ecological research: past and present. In: MiaO, S. et al. (Ed.). Real world ecology. Large-scale and long-term case studies and methods. New York: Springer Science, 2009. p. 1-18.

Miao, S. et al. (Ed.). Real world ecology. Large-scale and long-term case studies and methods. New York: Springer Science, 2009.

Mitman, G. The state of nature: ecology, community, and American social thought, 1900-1950. Chicago/London: University of Chicago Press, 1992.

Morey, M. Lectura de Foucault. Madrid: Taurus, 1986.

NúñEz, P. G. et al. Práctica científica y financiación. Un debate pendiente para la ecología. Ecología Austral, 19, p. 239-45, 2009 .

Odum, E. \& Barrett, G. W. Fundamentos de ecología. México: Cengage Learning, 2008 [1953].

OpdAM, P. Matapopulation theory and habitat fragmentation: a review of holarctic breeding bird studies. Landscape Ecology, 5, 2, p. 93-106, 1991.

Pasquo, F. M. Di. Experimentos, presupuestos epistémicos y “cientificidad” en la ecología: el caso de la macroecología. Filosofía e História da Biologia, 7, 1, p. 27-4,6, 2012.

PAsquo, F. M. DI et al. La ecología disciplinar y la intrusión de la problemática ambiental: hacia la "percepción de fenómenos globales”. Observatorio Medioambiental, 14, p. 21-39, 2011.

Pasquo, F. M. di \& Folguera, G. Crisis ambiental y ecología disciplinar: entre obstáculos epistemológicos y metodológicos. Epistemología e Historia de la Ciencia, Córdoba, 18, p. 208-15, 2012.

Passmore, J. La responsabilidad del hombre frente a la naturaleza. Madrid: Alianza, 1978.

Pengue, W. Fundamentos de economía ecológica. Buenos Aires: Kaicron, 2009.

Pickett, S. T. A.; Kolasa, J. \& Jones, C. G. Ecological understanding. New York: Elsevier, 2007.

Pozo, R. G. del. Estructuralismo y genealogía en la obra arqueológica de Michel Foucault. Sevilla: Universidad de Sevilla, 1986.

Prado, M. M. Apuntes sobre la reflexividad en el movimiento ecologista. Política y Sociedad, 23, p. 153$72,1996$.

Puebla, J. G. Cambio y persistencia en el espacio geográfico: consideraciones para la reflexión medioambiental. Observatorio Medioambiental, 2, p. 25-39, 1999.

Real, L. A. \& Brown, J. (Ed.). Foundations of ecology. Classic papers with commentaries. Chicago/London: The University of Chicago Press, 1991.

Resetarits, W. J. \& Bernardo, J. (Ed.). Experimental ecology. Issues and perspectives. Oxford: Oxford University Press, 1998.

SaArinen, E. (Ed.). Conceptual issues in ecology. Boston: Dordrecht Reidel, 1982.

Schneider, D. C. The rise of the concept of scale in ecology. BioScience, 51, 7, p. 545-54, 2001.

Sterelny, K. The elusive synthesis. In: Cuddington, K. \& Beisner, B. E. (Ed.). Ecological paradigms lost. Routes of theory change. New York: Elsevier Academic Press, 2005. p. 311-28.

Turner, M. G. et al. Landscape ecology in theory and practice: pattern and process. New York: Springer-Verlag, 2001.

VALDÉs, M. M. (Ed.). Naturaleza y valor. Una aproximación a la ética ambiental. México: Fondo de Cultura Económica, 2004: [1978].

Veyne, P. Un arqueólogo escéptico. In: Eribon, D. (Ed.). El infrecuentable Michel Foucault. Buenos Aires: Letra Viva, 2004. p. 23-87. 
UnA Historia de LA PRoblemática AMBiental...

Wiens, J. A. Spatial scaling in ecology. Functional Ecology, 3, 4, p. 385-97, 1989.

Wiens, J. A. et al. Integrating landscape structure and scale into natural resource management. In: Liv, J. \& TAYLOR, W. W. (Ed.). Integrating landscape ecology into natural resource management. Cambridge: Cambridge University Press, 2004. p. 23-67.

Wu, J. Scale and scaling: a cross-disciplinary perspective. In: Wu, J. \& Новвs, R. J. (Ed.). Key topics in landscape ecology. Cambridge: Cambridge University Press, 2007. p. 115-42.

Wu, J. \& Новвs, R. J. (Ed.). Key topics in landscape ecology. Cambridge: Cambridge University Press, 2007. Yustos, P. S. La conciencia ecológica. El espejo de una civilización suicida. Gazeta de Antropología, 25, 2, 2009. Disponible en: <http://digibug.ugr.es/html/10481/69 o1/G25_39Policarpo_Sanchez_Yustos. html>. Acceso en: 12 sep. 2013.

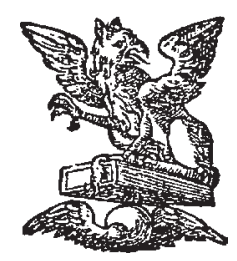

